\title{
An Analytical Study of a Tradeoff Between Transmission Power and FEC for TCP Optimization in Wireless Networks
}

\author{
Laura Galluccio, Giacomo Morabito, Sergio Palazzo
}

\begin{abstract}
It is well known that TCP has performance problems when wireless links are involved in the end-to-end connection. This is due to the high bit error rate characterizing wireless links. Appropriate power management and error correction can improve the link reliability observed by TCP and increase the throughput performance accordingly. In the literature, the effects of transmission power and error correction capability on TCP performance have been considered separately, so far. In this paper, a study of the tradeoff between power management and error correction is presented. To this end, an analytical framework to maximize a user satisfaction function, defined as the ratio between the TCP throughput and a cost function, is introduced. The proposed analytical framework does not depend on the specific wireless system and does not rely on any TCP throughput approximation formula. The benefits of joint power management and error control are demonstrated in several relevant case studies.
\end{abstract}

Key Words: Wireless Networks, TCP, Power Management, Forward Error Correction.

\section{INTRODUCTION}

It is well known in the literature that TCP has severe performance problems when wireless links are involved in the end-to-end connection [15]. In fact, factors such as interference, multipath fading, atmospheric conditions and possibly unpredictable user mobility cause link errors which may result in segment losses. The TCP sender interprets these segment losses as signal of network congestion and accordingly decreases the transmission rate. These transmission rate decreases are unnecessary and lead to resource inefficiency.

Two types of solutions to this problem have been proposed: Modifying TCP and Improving the Link Reliability observed by TCP [19].

Many TCP modifications have been introduced in literature, e.g., [2], [3], [4], [5], [7]. However, all of the solutions present problems related to reliability, scalability, security and backward compatibility.

Improving the link reliability observed by TCP seems more appealing because only modifications in the elements involved in the wireless communication are required [19]. This can be achieved either by means of error correcting protocols

This work was partially supported by MIUR under contract RAMON. Authors are with Dipartimento di Ingegneria Informatica e delle Telecomunicazioni, University of Catania, Catania (ITALY) (email: \{laura.galluccio, giacomo.morabito, sergio.palazzo \} @ diit.unict.it) such as Forward Error Correction (FEC) [6] or by setting the transmission power level appropriately [20]. However, the use of this type of solution causes additional costs. In fact, on the one hand, FEC introduces redundancy for error correction purposes and this redundancy utilizes scarce and costly wireless resources. On the other hand, increasing transmission power to decrease the bit error rate results in higher energy consumption and thus in shorter battery life.

The effects of FEC and power management on TCP performance have been studied separately in [6] and [20]. Little effort has been devoted so far to considering the effects of both FEC and power management at the same time.

In this paper we study the joint effects of FEC and power management on TCP performance and how they can be utilized for performance optimization purposes. To this end, we define a cost and a satisfaction function, where satisfaction is the ratio between throughput and cost. We develop a general analytical framework for maximization of the satisfaction function and derive an equation which relates the optimal redundancy ratio introduced by FEC and the optimal level of transmission power. The relationship is independent of the specific wireless system and does not rely on any approximation of the throughput formula. The analytical framework is applied to the most typical wireless communication scenarios to show the advantages of joint FEC and power management. Numerical results show that for performance maximization purposes an optimal tradeoff between FEC and power management exists.

The rest of the paper is organized as follows. In Section II we describe how the link reliability observed by TCP can be improved by using error correcting protocols and appropriate power management. In Section III we introduce an analytical framework for maximization of performance by means of joint error control and power management. The analytical framework is used in Section IV to demonstrate the advantages of joint error control and power management in three relevant scenarios. In Section V some conclusions are drawn.

\section{TeChniques to Improve the Link Reliability OBSERVED B Y TCP}

\section{A. Forward Error Correction (FEC)}

Forward Error Correction (FEC) is based on adding redundancy to data being transmitted. This redundancy is encoded and used to correct possible transmission errors due to medium characteristics. The information sequence is divided 
into blocks of length $K$ bits. FEC maps each of these blocks into a codeword of length $N$, with $N>K$. Accordingly, $2^{K}$ binary sequences of length $N$, called codewords, are defined. The code rate, $R_{c}$, is defined as

$$
R_{c}=K / N
$$

We also define the redundancy ratio, $x$, as the ratio between the amount of redundancy introduced by FEC, $(N-K)$, and the block length, $K$, i.e.,

$$
x=(N-K) / K
$$

From the definition of the redundancy ratio, $x$, it follows that the code rate is given by:

$$
R_{c}=1 /(1+x)
$$

Let us denote the $i$-th codeword as $\mathbf{c}_{\mathbf{i}}$, where $1 \leq i \leq$ $2^{K}$. The Hamming distance, $d^{H}$, between two code words $\mathbf{c}_{\mathbf{i}}$ and $\mathbf{c}_{\mathbf{j}}$ is defined as the number of bits by which the two codewords differ from each other [17]. The Hamming distance is an important feature of FEC schemes because it determines the correction capability of a coding scheme.

The coding gain, $G_{\text {coding }}$ is defined as [17]

$$
G_{\text {Coding }}=d_{\min }^{H} \cdot R_{c}
$$

where $d_{\min }^{H}$ is the minimum Hamming distance. As an example, $d_{\min }^{H}=(N-K+1)$ if Reed-Solomon encoding [18] is applied $^{1}$, and therefore the coding gain is given by

$$
G_{\text {Coding }}=\frac{K \cdot x+1}{1+x}
$$

FEC techniques allow retransmissions to be avoided and consequently reduce delivery delay. For this reason, they are suitable for real-time traffic with stringent time requirements. Recently FEC techniques have been applied to non-real-time (TCP) traffic as well. In fact, the use of FEC reduces the number of segment losses observed by TCP and, accordingly, the number of unnecessary transmission rate decreases, thus improving wireless resource efficiency [8].

However, FEC techniques also imply bandwidth waste, which may turn into an overall decrease in wireless resource efficiency. Therefore, the amount of redundancy to be introduced must be set very carefully so as to maximize resource efficiency. In fact, an optimal value exists and any increase in redundancy beyond this value results in a deterioration of throughput performance. In [6] Barakat and Altman derive an analytical expression for throughput in TCP connections, depending on FEC redundancy. By using this analytical expression the optimal amount of FEC can be evaluated according to the current channel conditions and some TCP parameters.

\footnotetext{
${ }^{1} \mathrm{~A}$ burst of errors results in few symbol errors if Reed-Solomon encoding is applied. For this reason this encoding scheme is very convenient for wireless and satellite communications in which errors appear in bursts.
}

\section{B. Power Management}

Link reliability can also be improved by increasing the transmission power. In fact, the bit error probability, $P_{e}$, decreases when the ratio $\left(E_{b} / N_{0}\right)$ increases, where $E_{b}$ is the received energy per bit and $N_{0}$ is the noise power spectral density. Note that the relationship between the bit error probability, $P_{e}$, and the ratio $\left(E_{b} / N_{0}\right)$ is a function of the modulation technique.

Let $y$ represent the transmission power. The received energy per bit, $E_{b}$, is given by:

$$
E_{b}=A \cdot y / B
$$

In the above equation, $B$ is the link bandwidth and $A$ is the attenuation.

Increasing the transmission power improves TCP performance but causes greater energy consumption, which results in shorter battery life for portable devices. In addition to this, increasing the power level aggravates the interference for other neighboring communications.

A detailed study of the impact of transmission power on TCP performance is given in [20].

\section{ThE ANALYTICAL FRAMEWORK}

We define the satisfaction function, $\gamma$, as the ratio between the throughput, $\sigma$, and the $\operatorname{cost} c$, i.e., $\gamma=\sigma / c$. Evaluation of the cost function, $c$, and throughput, $\sigma$, is made in Sections III-A and III-B, respectively. In Section III-C, we develop the general analytical framework for the maximization of $\gamma$.

\section{A. Cost Evaluation}

Consider the transfer of $S$ segments each of $M S S$ bits in size. The cost of the transfer depends on the transmission power and the amount of redundancy introduced. More specifically, we consider two cost terms: a term which takes energy consumption into account, and a term which considers the amount of wireless resources employed.

If the redundancy ratio introduced by FEC is $x$, then $[S$. $M S S \cdot(1+x)]$ bits must be transmitted in order to deliver $S$ segments of $M S S$ bits each. Accordingly, the cost of the resources required to complete the transfer is given by:

$$
c_{\text {Resources }}=k_{\text {Resources }} \cdot S \cdot M S S(1+x)
$$

where $k_{\text {Resources }}$ (expressed in $\left[b i t^{-1}\right]$ ) is a constant which represents the cost of the bandwidth resources required to transfer a bit. Given that the energy transmitted per bit is given by $(y / B)$, the energy consumption required to complete the transfer is $[S \cdot M S S \cdot(1+x) \cdot y / B]$. As a result, if $k_{\text {Energy }}$ (expressed in $\left[J_{\text {oul }} e^{-1}\right]$ ) represents the cost of a unit of energy, then the total energy cost is:

$$
c_{\text {Energy }}=k_{\text {Energy }} \cdot S \cdot M S S \cdot(1+x) \cdot y / B
$$

Accordingly, when the redundancy is $x$ and the energy transmitted per bit is $y$, the cost of the transfer can be evaluated as 


$$
\begin{aligned}
& c(x, y)=c_{\text {Energy }}+c_{\text {Resources }}= \\
& \quad=S \cdot M S S \cdot(1+x) \cdot\left(k_{\text {Energy }} \cdot y / B+k_{\text {Resources }}\right)
\end{aligned}
$$

Observe that the constants $k_{\text {Resources }}$ and $k_{\text {Energy }}$ depend on many factors such as user preferences, the type of terminal and the battery status. In most cases, wireless devices are supplied by batteries which have limited energy capabilities.

\section{Example}

As an example we evaluate the relationship between $k_{\text {Resources }}$ and $k_{\text {Energy }}$ if we assume that the cost increase due to a percentage variation of $\Delta$ in the energy transmitted per bit, $y$, is higher than the cost increase due to the same percentage variation in the redundancy $x$. Let $\Delta c_{x}$ be the cost increase when the redundancy increases by $(\Delta \cdot x)$, i.e.,

$$
\begin{aligned}
& \Delta c_{x}=c(x \cdot(1+\Delta), y)-c(x, y)= \\
& \quad=S \cdot M S S \cdot \Delta \cdot x \cdot\left(\frac{k_{\text {Energy }}}{B} \cdot y+k_{\text {Resources }}\right)
\end{aligned}
$$

and let $\Delta c_{y}$ be the cost increase when the energy transmitted per bit increases by $(\Delta \cdot y)$, i.e.,

$$
\begin{aligned}
\Delta c_{y}=c & (x, y \cdot(1+\Delta))-c(x, y)= \\
& =S \cdot M S S \cdot \Delta \cdot \frac{k_{\text {Energy }}}{B} \cdot y \cdot(1+x)
\end{aligned}
$$

The condition $\Delta c_{y}>\Delta c_{x}$ implies that the following relationship must hold:

$$
\frac{k_{\text {Energy }}}{k_{\text {Resources }}}>\frac{x}{y} \cdot B
$$

In order to simplify the notation in the rest of the paper we define:

$$
k_{1}=S \cdot M S S \cdot k_{\text {Energy }} / B
$$

and

$$
k_{2}=S \cdot M S S \cdot k_{\text {Resources }}
$$

Accordingly, the cost function is given by:

$$
c(x, y)=(1+x) \cdot\left(k_{1} \cdot y+k_{2}\right)
$$

and eq. (12) can be written as:

$$
\frac{k_{1}}{k_{2}}>\frac{x}{y}
$$

\section{B. TCP Throughput Evaluation}

Let $b$, with $b \leq B$, be the available bit rate for a given TCP connection. The throughput formulas proposed in the literature can be roughly represented as follows:

$$
\sigma=\frac{b}{1+x} \cdot f\left(R T T, P_{\text {Loss }}\right)
$$

where

- $f(\cdot, \cdot)$ is an appropriate function which describes the impact of the round trip time and link errors on TCP throughput performance. Several of these functions have been proposed in the literature, e.g., [12], [15], [16], [20]. Our analysis is independent of the specific $f(\cdot, \cdot)$.
- $R T T$ is the round trip time. Observe that the dependence of $R T T$ on $x$ and $y$ can be neglected.

- $P_{\text {Loss }}$ is the probability that a TCP segment is discarded because of link errors in the wireless channel. Assuming that losses of blocks are i.i.d., the probability $P_{\text {Loss }}$ can be evaluated as

$$
P_{\text {Loss }}=1-\left(1-P_{\text {Block }}\right)^{M S S / K}
$$

where $P_{\text {Block }}$ is the block error probability at the output of the decoder. The probability $P_{\text {Block }}$ is related to the redundancy ratio $x$ and the transmission power $y$ as follows [17]:

$$
P_{\text {Block }}=h\left(y \cdot G_{\text {Coding }}\right)
$$

where the expression of $h(\cdot)$ depends on the modulation technique used [17]. Replacing $G_{\text {Coding }}$ with the expression provided in eq. (5), which is valid when ReedSolomon encoding is used, we obtain ${ }^{2}$

$$
P_{\text {Block }}=h\left(y \cdot \frac{K \cdot x+1}{x+1}\right)
$$

The expression $P_{\text {Block }}$ is provided in [17] for several relevant examples. We will give some of them in Section IV.

In summary, the throughput given in eq. (17) can be written as a function of $x$ and $y$ as follows:

$$
\sigma(x, y)=\frac{b}{1+x} \cdot f(R T T, g(h(\phi(x, y))))
$$

where:

- $f\left(R T T, P_{\text {Loss }}\right)$ is the function which approximates the impact of the round trip time, $R T T$, and the probability that a segment is lost due to link errors, $P_{\text {Loss }}$.

- $g\left(P_{\text {Block }}\right)$ is defined as

$$
g\left(P_{\text {Block }}\right)=1-\left(1-P_{\text {Block }}\right)^{M S S / K}
$$

and represents the relationship between the segment loss probability, $P_{\text {Loss }}$, and the block error probability, $P_{\text {Block }}$.

- $h(z)$ is the equation which provides the block error probability as a function of the product between the transmission power, $y$, and the coding gain, $G_{\text {Coding }}$.

- $\phi(x, y)$ is defined as

$$
\phi(x, y)=y \cdot \frac{K \cdot x+1}{x+1}
$$

and represents the product between the transmission power and the coding gain.

\footnotetext{
${ }^{2}$ Note that using different coding schemes we can obtain similar expressions for the coding gain, $G_{\text {Encoding. }}$.
} 


\section{Satisfaction Function Maximization}

Let us define the satisfaction function $\gamma(x, y)$ as follows:

$$
\gamma(x, y)=\frac{\sigma(x, y)}{c(x, y)}
$$

From eq. (21) and eq. (15) it follows that

$$
\gamma(x, y)=\frac{b \cdot f(R T T, g(h(\phi(x, y))))}{(1+x)^{2} \cdot\left(k_{1} \cdot y+k_{2}\right)}
$$

Now we want to evaluate the values of $x$ and $y$ which maximize the function $\gamma(x, y)$. To do so, we need to find the solutions of the following system:

$$
\begin{aligned}
& \frac{\partial \gamma}{\partial x}(x, y)=0 \\
& \frac{\partial \gamma}{\partial y}(x, y)=0
\end{aligned} .
$$

Since the expression of the satisfaction function depends on the specific modulation scheme used, it is impossible to find a general closed solution for the system in eq. (26). For this reason, we proceed by steps: first, we find a relationship between the values of $x$ and $y$ which maximize the satisfaction function. Then, we substitute this relationship in the satisfaction function $\gamma(x, y)$, which therefore becomes a function of one variable. Finally, we find the maximum numerically.

Substituting eq. (25) in eq. (26) we obtain:

$$
\begin{aligned}
& \frac{\partial F}{\partial x}(x, y) \cdot(1+x)=2 \cdot F(x, y) \\
& \frac{\partial F}{\partial y}(x, y) \cdot\left(k_{1} \cdot y+k_{2}\right)=k_{1} \cdot F(x, y)
\end{aligned}
$$

where $F(x, y)$ is defined as:

$$
F(x, y)=f(R T T, g(h(\phi(x, y))))
$$

From eq. (27), it follows that:

$$
2 \cdot \frac{\partial F}{\partial y}(x, y) \cdot\left(k_{1} \cdot y+k_{2}\right)=k_{1} \cdot \frac{\partial F}{\partial x}(x, y) \cdot(1+x)
$$

The derivatives in eq. (29) can be evaluated as:

$$
\begin{gathered}
\frac{\partial F}{\partial x}(x, y)=\frac{\partial f}{\partial P_{\text {Loss }}}(R T T, g(h(\phi(x, y)))) . \\
g^{\prime}(h(\phi(x, y))) \cdot h^{\prime}(\phi(x, y)) \cdot \frac{\partial \phi}{\partial x}(x, y)
\end{gathered}
$$

and

$$
\begin{gathered}
\frac{\partial F}{\partial y}(x, y)=\frac{\partial f}{\partial P_{\text {Loss }}}(R T T, g(h(\phi(x, y)))) . \\
g^{\prime}(h(\phi(x, y))) \cdot h^{\prime}(\phi(x, y)) \cdot \frac{\partial \phi}{\partial y}(x, y)
\end{gathered}
$$

Substituting eqs. (30) and (31) in eq. (29), we obtain:

$$
2 \cdot \frac{\partial \phi}{\partial y}(x, y) \cdot\left(k_{1} \cdot y+k_{2}\right)-k_{1} \cdot \frac{\partial \phi}{\partial x}(x, y) \cdot(1+x)=0
$$

where $\frac{\partial \phi}{\partial y}(x, y)$ and $\frac{\partial \phi}{\partial x}(x, y)$ can be obtained by differentiating eq. (23), i.e.,

$$
\frac{\partial \phi}{\partial x}(x, y)=\frac{(K-1) \cdot y}{(1+x)^{2}}
$$

and

$$
\frac{\partial \phi}{\partial y}(x, y)=\frac{K \cdot x+1}{(1+x)}
$$

Eqs. (33) and (34) can be used in eq. (32) to obtain:

$$
y=\frac{2 \cdot k_{2}}{k_{1}} \cdot \frac{1+K \cdot x}{K-3-2 \cdot K \cdot x}
$$

Note that eq. (35), specifying the function which relates the optimal values of the redundancy ratio, $x$, and transmission power, $y$, is in closed form and does not depend on $f(\cdot, \cdot)$, $g(\cdot)$ and $h(\cdot)$. Therefore, the relationship between the optimal values of $x$ and $y$ is independent of the specific modulation adopted and does not rely on any TCP throughput approximation. In Section IV, we will need the function $\psi(x)$ defined as follows:

$$
\psi(x)=\frac{2 \cdot k_{2}}{k_{1}} \cdot \frac{1+K \cdot x}{K-3-2 \cdot K \cdot x}
$$

It is worth noting that since the optimal value of the transmission power must be positive, according to eq. (35), the optimal value of the redundancy ratio $x$ is upper-bounded and is to be searched for in the interval $[0,(1 / 2)-3 /(2 \cdot K)[$. In Figure 1 we show the dependence of $y$ (divided by the constant factor $\left[2 \cdot k_{2} / k_{1}\right]$ ) on $x$ for different values of $K$ as provided in eq. (35).

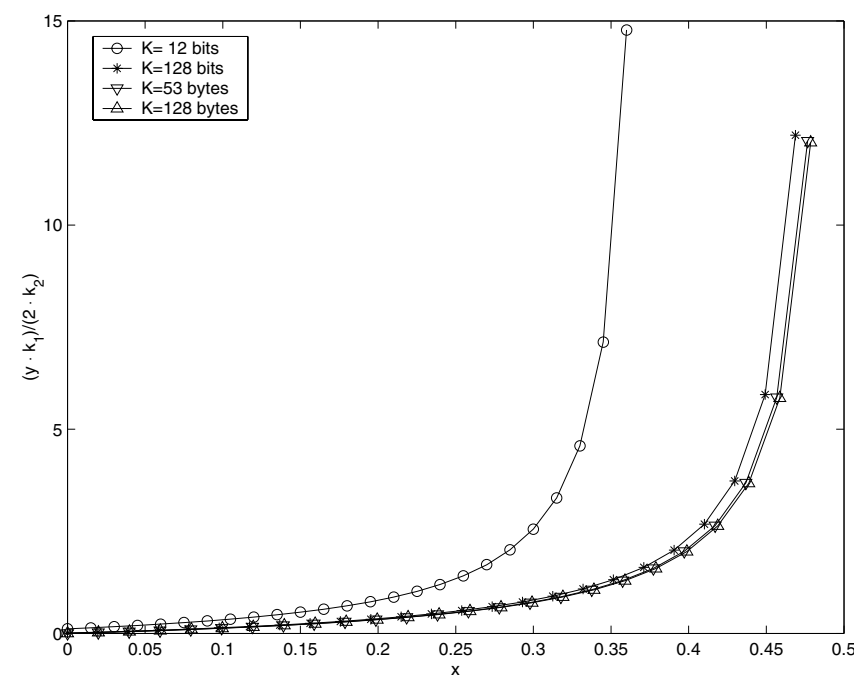

Fig. 1. $\left[\frac{y \cdot k_{1}}{2 \cdot k_{2}}\right]$ as given in eq. (35) vs. $x$ for different values of $K$.

Now, let us define $\tilde{\gamma}(x)$ as the function obtained by replacing eq. (35) in eq. (24), i.e.,

$$
\tilde{\gamma}(x)=b \cdot(K-3-2 \cdot K \cdot x) \cdot \frac{f(R T T, g(h(\tilde{\phi}(x))))}{(1+x)^{2} \cdot k_{2} \cdot(K-1)}
$$

where $\tilde{\phi}(x)$ has been obtained by replacing eq. (35) in eq. (23), i.e.,

$$
\tilde{\phi}(x)=\frac{2 \cdot k_{2}}{k_{1}} \cdot \frac{(K \cdot x+1)^{2}}{(1+x) \cdot(K-3-2 \cdot K \cdot x)}
$$

Observe that $\tilde{\gamma}(x)$ is only a function of one variable and therefore its maximum in the interval

$$
0 \leq x<\frac{1}{2}-\frac{3}{2 \cdot K}
$$


can be found very easily by means of numerical calculations. The optimal values of the amount of redundancy, $x_{\mathrm{opt}}$, and transmission power, $y_{\mathrm{opt}}$, can be calculated as follows:

- $x_{\mathrm{opt}}$ is the value of $x$ which maximizes $\tilde{\gamma}(x)$ as defined in eq. (37).

- $y_{\text {opt }}$ can be evaluated by replacing $x$ with $x_{\text {opt }}$ in eq. (35).

\section{CAse Study}

In this section we apply the analytical framework developed in the previous section to three relevant examples of numerical modulation techniques, i.e.,

1) Gaussian M-ary Shift Keying (GMSK), which is used in the General Packet Radio Service (GPRS).

2) Differentiated Binary Phase Shift Keying (DBPSK), which is used in IEEE 802.11.

3) Gaussian Frequency Shift Keying (GFSK), which is used in Bluetooth.

\section{A. TCP Throughput Approximation Formula}

In the literature several formulas have been proposed to approximate the behavior of TCP throughput [12], [15], [16], [20]. Here we will use the heuristic formula proposed in [20] which is the most accurate for wireless communications. According to it, the throughput of a TCP connection is given by [20]

$$
\sigma \approx \sigma_{0} \cdot \begin{cases}\frac{10^{\epsilon \cdot \ln \left(\frac{1}{\tilde{\xi}-\xi_{s}}-1\right)}}{10^{\epsilon \cdot \ln \left(\frac{1}{\tilde{\xi}-\xi_{s}}-1\right)}+1} & \text { if } \tilde{\xi}>\xi_{s} \\ 1 & \text { if } \tilde{\xi} \leq \xi_{s}\end{cases}
$$

where:

- $\sigma_{0}$ is the average throughput for error-free links. If the available bit rate is $b, \sigma_{0}$ can be calculated as $\sigma_{0}=b /(1+$ $x)$.

- $\tilde{\xi}=1+\left[\log _{10}\left(P_{\text {Loss }}\right) / 3\right]$

- $\epsilon=1.3$

- $\xi_{s}=1 /\left(\theta \cdot f_{d}+\beta\right)-\kappa$. Note that $f_{d}$ is the Doppler frequency used to model the Rayleigh fading [20], whereas $\theta, \beta$ and $\kappa$ are constants, i.e., $\theta=1.39, \beta=2.78$ and $\kappa=0.03$. A typical value for $f_{d}$ is $f_{d}=6 \mathrm{~Hz}$.

It is worth noting that the throughput expression given in eq. (40) is a heuristic function obtained by fitting the simulation curves. Thus, the involved constants do not have specific physical meaning, but satisfy the condition of giving an analytical expression for throughput which is reasonably close to the actual points obtained by simulation.

Accordingly, the function $f\left(R T T, P_{\text {Loss }}\right)$ is given by:

$$
f\left(R T T, P_{\text {Loss }}\right)= \begin{cases}\frac{10^{\epsilon \cdot \ln \left(\frac{1}{\tilde{\xi}-\xi_{s}}-1\right)}}{10^{\epsilon \cdot \ln \left(\frac{1}{\tilde{\xi}-\xi_{s}}-1\right)}+1} & \text { if } \tilde{\xi}>\xi_{s} . \\ 1 & \text { if } \tilde{\xi} \leq \xi_{s} .\end{cases}
$$

\section{B. Gaussian M-ary Shift Keying (GMSK)}

If GMSK is used, the bit error probability, $P_{e}$, is given by [17]:

$$
P_{e}=\frac{1}{2} \cdot \operatorname{erfc}\left(\sqrt{\frac{\alpha \cdot A}{N_{O} \cdot \Delta F} \cdot y \cdot \frac{K \cdot x+1}{x+1}}\right)
$$

where:

- $\operatorname{erfc}(u)$ is the Complementary Error Function and is defined as

$$
\operatorname{erfc}(u)=\frac{2}{\sqrt{\pi}} \cdot \int_{u}^{\infty} \exp \left(v^{2}\right) d v
$$

- $\alpha$ is a constant term which depends on the transmission bandwidth and the bit transmission time. In GPRS systems $\alpha=0.8$.

- $A$ is the attenuation which takes into consideration many features, mainly related to atmospheric phenomena, which contribute to the degradation of the signalto-noise ratio. Those features are the free-space loss attenuation, $A_{F S}$, the atmospheric gases attenuation, $A_{A}$, the attenuation due to the rain, $A_{\text {rain }}$, and the one due to the scintillation phenomenon, $A_{\text {scint }}$. Therefore, the expression for the attenuation $A$ can be written as follows:

$$
\begin{aligned}
A & =A_{F S} \cdot A_{A} \cdot A_{\text {rain }} \cdot A_{\text {scint }}= \\
& =\frac{\left(\lambda^{2}\right)}{(4 \cdot \pi \cdot d)^{2}} \cdot A_{A} \cdot A_{\text {rain }} \cdot A_{\text {scint }}
\end{aligned}
$$

Observe that, in the following case study we will consider only the free-space loss contribution to the attenuation.

- $N_{0}$ is the noise power spectral density, which is assumed to be constant and equal to $1.379 \cdot 10^{-20}[\mathrm{~W} / \mathrm{Hz}]$.

- $\Delta F$ is the size of the frequency band used. We assume that $\Delta F=25 \mathrm{MHz}$ as in GPRS.

As a consequence, the function $h(z)$ is defined as:

$$
h(z)=1-\left[1-\frac{1}{2} \cdot \operatorname{erfc}\left(\sqrt{\frac{\alpha \cdot A}{N_{O} \cdot \Delta F} \cdot z}\right)\right]^{K}
$$

We assume that the block size is $K=260$ bits, the TCP segment size is $M S S=128$ bytes and the bandwidth available for the TCP connection is $b=9.6 \mathrm{Kbps}$.

In Figures 2, 3 and 4 we study the effects of error correction and transmission power on link reliability and TCP performance in terms of throughput and the satisfaction function. More specifically, we show:

- Figure 2: The segment error probability, $P_{\text {Loss }}$, vs. the redundancy ratio, $x$, and transmission power, $y$.

- Figure 3: The TCP throughput, $\sigma(x, y)$, evaluated using eq. (40), vs. the redundancy ratio, $x$, and the transmission power, $y$.

- Figure 4: The satisfaction function, $\gamma(x, y)$, evaluated assuming $k_{1} / k_{2}=100$ and $k_{2}=1$, vs. the redundancy ratio, $x$, and the transmission power, $y$. 


\begin{tabular}{||l|l|l|l||}
\hline \hline Modulation & GMSK & DBPSK & GFSK \\
\hline Example & GPRS & IEEE 802.11 & Bluetooth \\
\hline$K$ & 260 bits & 12 bits & 625 bits \\
\hline$M S S$ & 128 bytes & 128 bytes & 128 bytes \\
\hline$\Delta F$ & $25 \mathrm{MHz}$ & $83 \mathrm{MHz}$ & $1 \mathrm{MHz}$ \\
\hline$N_{0}$ & $1.379 \mathrm{e}-20$ & $1.379 \mathrm{e}-20$ & $1.379 \mathrm{e}-20$ \\
\hline$b$ & $9.6 \mathrm{Kbps}$ & $128 \mathrm{Kbps}$ & $700 \mathrm{Kbps}$ \\
\hline$k_{1} / k_{2}$ & $10,100,1000$ & 100 & 1000 \\
\hline$d$ & $0-500 \mathrm{~m}$ & $0-150 \mathrm{~m}$ & $0-100 \mathrm{~m}$ \\
\hline \hline
\end{tabular}

TABLE I

Case Study Parameters.

The numerical choice of $\frac{k_{1}}{k_{2}}$ can be explained considering eq. (16). Since $0 \leq x \leq 1$ and $y$ is typically in the order of 10 $\mathrm{mW}$, it can be derived that $0<\frac{x}{y}<100$. So in order to satisfy eq. (16), we have chosen $\frac{k_{1}}{k_{2}}=100$.

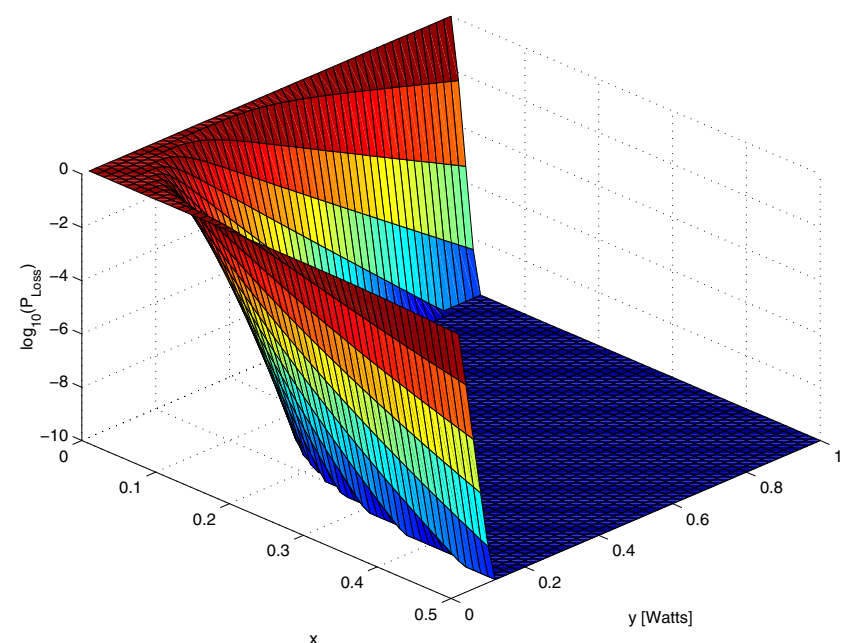

Fig. 2. Behavior of the segment error probability, $P_{\text {Loss }}$, vs. the redundancy ratio, $x$, and the transmission power, $y$.

Obviously, in Figure 2 the packet error probability always decreases as the redundancy ratio, $x$, and the transmission power, $y$, increase. In Figure 3, for any value of the redundancy ratio, $x$, the throughput is a non-decreasing function of the transmission power, $y$. This is obvious because the increase in the transmission power $y$ involves an improvement in the link reliability. In general a threshold value, $y_{T h}$, for the transmission power can be defined, such that:

- If $y<y_{T h}$ : a threshold value for the redundancy ratio $x_{T h}(y)$ can be observed. If $x<x_{T h}(y)$ the throughput increases as $x$ increases. This is because an increase in the redundancy ratio results in an improvement in the link reliability and thus a throughput increase. Otherwise, if $x \geq x_{T h}(y)$, the throughput decreases as $x$ increases. This is because the redundancy increase does not result in a significant increase in the link reliability and there-

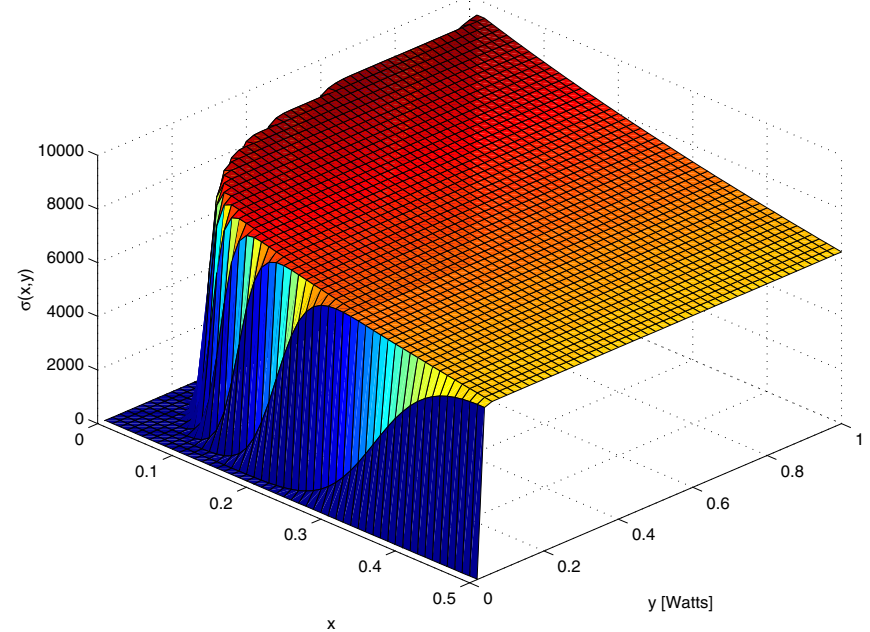

Fig. 3. Behavior of the throughput, $\sigma(x, y)$, vs. the redundancy ratio, $x$, and the transmission power, $y$.

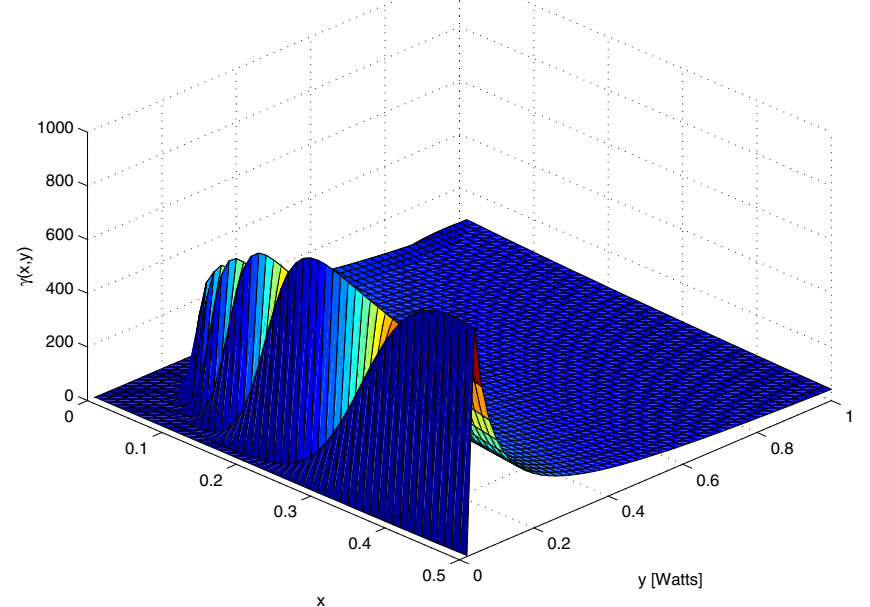

Fig. 4. Behavior of the satisfaction function, $\gamma(x, y)$, evaluated assuming $k_{1} / k_{2}=100$ and $k_{2}=1$, vs. the redundancy ratio, $x$, and the transmission power, $y$. 
fore, further redundancy only results in further overhead. Obviously the value of $x_{T h}(y)$ depends on $y$ : the higher $y$, the lower $x_{T h}(y)$.

- If $y \geq y_{T h}$ : an increase in the redundancy ratio always results in a decrease in the TCP throughput performance. This is because the transmission power is such that link reliability is high even if no error correction scheme is used. Accordingly, an increase in the redundancy ratio, $x$, only results in a throughput decrease.

The value of the threshold $y_{T h}$ depends on several factors, i.e., the modulation technique, the block size $K$, the segment size $M S S$, the attenuation, $A$, etc. In Figure 3 , the value of $y_{T h}$ is equal to $y_{T h}=0.6 \mathrm{~W}$. In Figure 4 , the satisfaction function $\gamma(x, y)$ increases as the redundancy ratio, $x$, and the transmission power, $y$, increase until they reach the values $x_{\mathrm{opt}}$ and $y_{\mathrm{opt}}$, respectively. This is because the throughput increase is more significant than the cost increase. Note that $x_{\mathrm{opt}}$ and $y_{\text {opt }}$ are the optimal values of $x$ and $y$, i.e., those which maximize the satisfaction function. In Figure 4, the optimal values are $x_{\mathrm{opt}}=0.4$ and $y_{\mathrm{opt}}=43 \mathrm{~mW}$. On the contrary, for high values of $x$ and $y$, i.e., $x>x_{\mathrm{opt}}$ and $y>y_{\mathrm{opt}}$, the satisfaction function, $\gamma(x, y)$ decreases when $x$ and $y$ increase. Figures 2, 3 and 4 were obtained assuming that the distance between the transmitter and receiver is $d=150 \mathrm{~m}$.

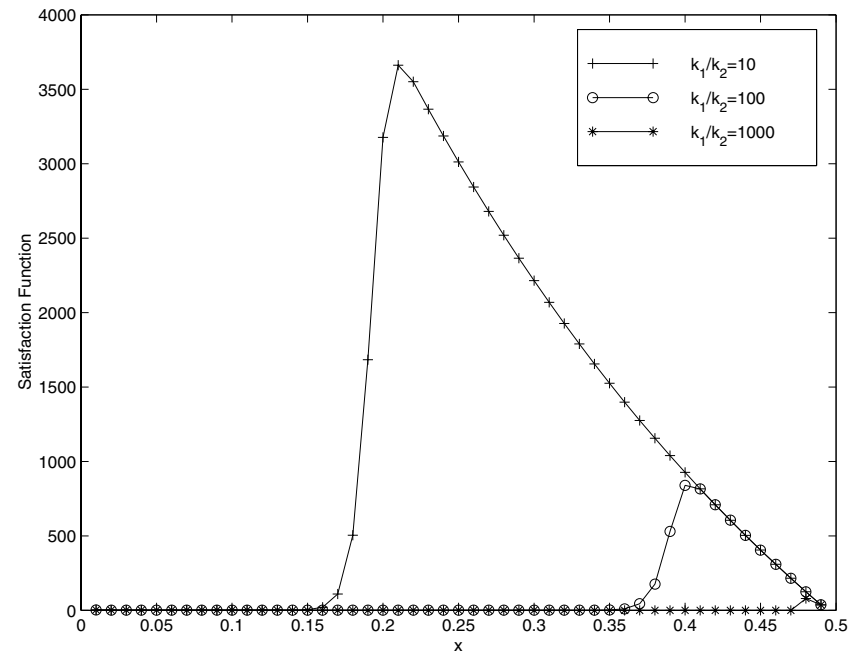

Fig. 5. Satisfaction function, $\tilde{\gamma}(x)$, vs. the redundancy ratio $x$ for different values of the ratio $k_{1} / k_{2}$.

Now we will evaluate the optimal values of the redundancy ratio, $x$, and transmission power, $y$, using the analytical results obtained in Section III-C. In Figure 5 we show the function $\tilde{\gamma}(x)$ given in eq. (37) for different values of the ratio between $k_{1}$ and $k_{2}$. The optimum value of the redundancy ratio $x_{\mathrm{opt}}$ is the one which maximizes the function $\tilde{\gamma}(x)$ shown in Figure 5 . It can easily be determined by numerical analysis of $\tilde{\gamma}(x)$, whereas the optimum value of the transmission power $y_{\mathrm{opt}}$ can be obtained by replacing $x$ with $x_{\text {opt }}$ in eq. (35). As an example, if the ratio $\left(k_{1} / k_{2}\right)$ is equal to 100 , the values of

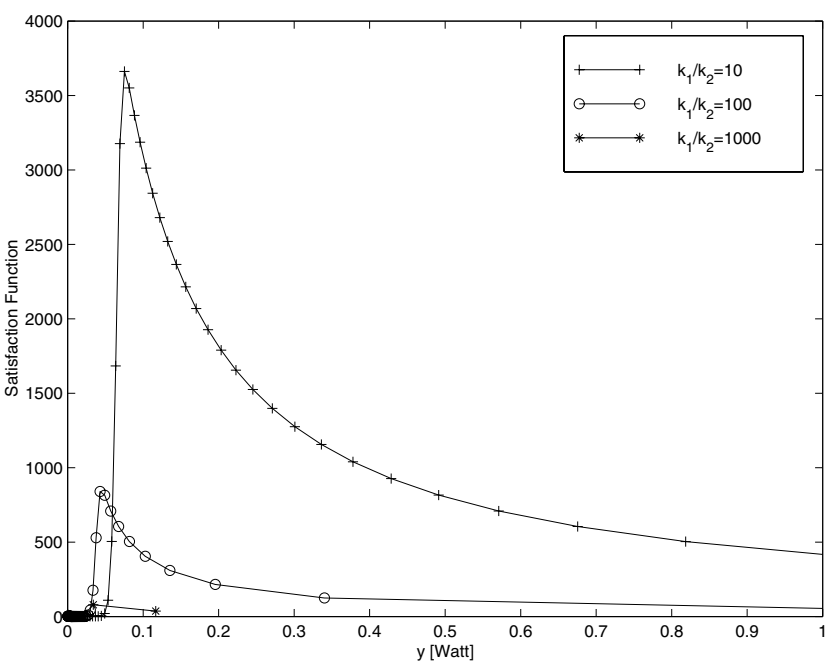

Fig. 6. Satisfaction function, $\tilde{\gamma}\left(\psi^{-1}(x)\right)$, vs. the transmission power, $y$, for different values of the ratio $k_{1} / k_{2}$.

$x_{\text {opt }}$ and $y_{\text {opt }}$ are equal to 0.4 and $43 \mathrm{~mW}$, respectively ${ }^{3}$.

In Figure 5, we observe that the optimal value of the redundancy ratio, $x_{\mathrm{opt}}$, increases as the ratio $\left(k_{1} / k_{2}\right)$ increases. On the contrary, the value of $y_{\text {opt }}$ decreases as $\left(k_{1} / k_{2}\right)$ increases. This is because, as $\left(k_{1} / k_{2}\right)$ increase, the cost of the energy becomes higher than the cost of wireless bandwidth resources. Therefore, it is better to improve the link reliability by introducing redundancy rather than increasing the transmission power. This is shown in Figure 6, where we show the value of $\tilde{\gamma}\left(\psi^{-1}(y)\right)$ vs. the transmission power $y$. Note that $\psi^{-1}(y)$ is the inverse of the function, $\psi(x)$ which was defined in eq. (36).

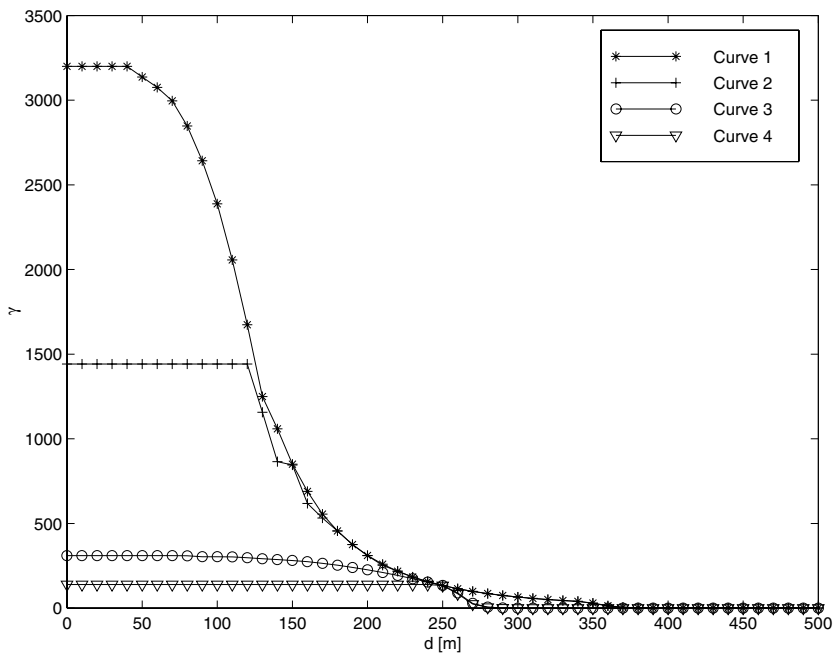

Fig. 7. Maximum satisfaction function, $\gamma$, vs. the distance $d$ between the transmitter and the receiver.

${ }^{3}$ Observe that the optimal values $x_{\mathrm{opt}}$ and $y_{\mathrm{opt}}$ depend on the ratio between $k_{1}$ and $k_{2}$ and not on their specific values. 
Now we want to evaluate the advantages of joint FEC and power management. To this end, in Figure 7 we show the maximum value of the satisfaction function, i.e., $\gamma\left(x_{\mathrm{opt}}, y_{\mathrm{opt}}\right)$, vs. the distance between transmitter and receiver $d$. For the sake of comparison, in the above figure, four curves are depicted:

1) Curve 1: The maximum value of the satisfaction function $\gamma\left(x_{\mathrm{opt}}, y_{\mathrm{opt}}\right)$.

2) Curve 2: The maximum value of the satisfaction function once the transmission power, $y$, has been set to $\bar{y}$, i.e.,

$$
\max _{x}[\gamma(x, \bar{y})]
$$

where $\bar{y}$ is the optimal value of $y$ when $d=250 \mathrm{~m}$.

3) Curve 3: The maximum value of the satisfaction function once the redundancy ratio, $x$, has been set to $\bar{x}$, i.e.,

$$
\max _{y}[\gamma(\bar{x}, y)]
$$

where $\bar{x}$ is the optimal value of $x$ when $d=250$ $\mathrm{m}$ which is the average value of the distance in the considered transmission range.

4) Curve 4: The value of $\gamma(\bar{x}, \bar{y})$ vs. the distance between transmitter and receiver, $d$.

All the curves in Figure 7 exhibit non-increasing behavior. This is because, as the distance increases, higher correcting capabilities and transmission power are required in order to obtain satisfactory TCP throughput, and the communication thus has a higher cost. However, we can observe that the satisfaction value achieved by joint FEC and power management is always the highest. Also, we note that with high values for the distance $d$ the differences between Curve 1 and the other curves is slight. This is because when the distance increases, higher transmission power is required to increase the TCP throughput and therefore for long distances the cost becomes high whatever approach is used.

\section{Differentiated Binary Phase Shift Keying (DBPSK)}

If DBPSK is utilized, then the block error probability, $P_{\text {Block }}$ at the output of the FEC decoder is [17]

$$
P_{\text {Block }}=1-\left[1-\frac{1}{2} \cdot \exp \left(-\frac{A \cdot y}{N_{0} \cdot \Delta F} \cdot \frac{K \cdot x+1}{1+x}\right)\right]_{(48)}^{K}
$$

Accordingly, the function $h(z)$ is defined as

$$
h(z)=1-\left[1-\frac{1}{2} \cdot \exp \left(-\frac{A \cdot z}{N_{0} \cdot \Delta F}\right)\right]^{K}
$$

In order to show the advantages of joint management of FEC and transmission power, in Figure 8 we give the optimal values of the satisfaction function, $\gamma$, vs. the distance between the transmitter and the receiver. In the sake of comparison similarly to Figure 7, four curves are shown in Figure 8. Their meaning was described in Section IV-B. Observe that Figure 8 was obtained using the parameters given in Table I.

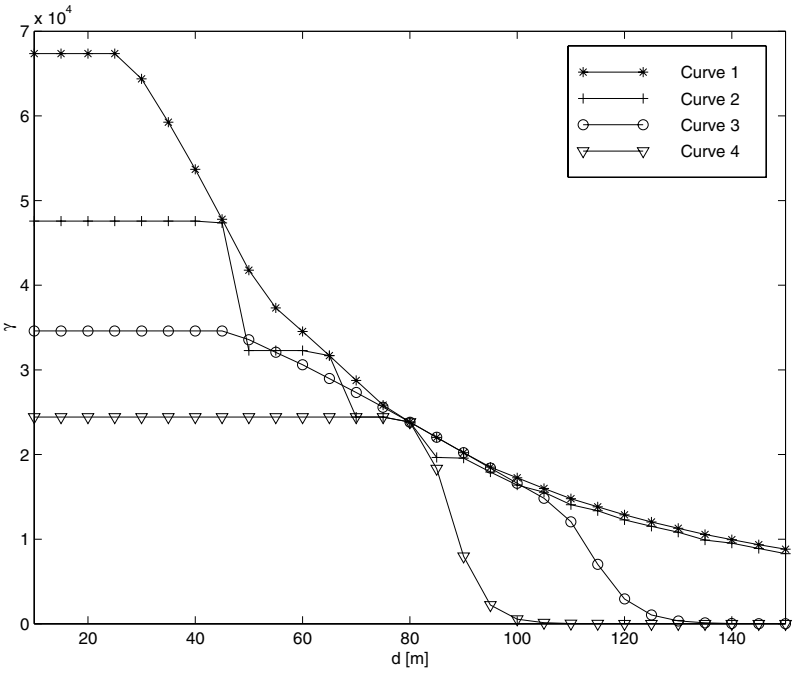

Fig. 8. Maximum satisfaction function, $\gamma$, vs. the distance $d$ between the transmitter and the receiver.

\section{Gaussian Frequency Shift Keying (GFSK)}

If GFSK is utilized, then the block error probability, $P_{B l o c k}$ at the output of the FEC decoder is [17]

$P_{\text {Block }}=1-\left[1-\frac{1}{2} \cdot \exp \left(-\frac{1}{2} \cdot \frac{A \cdot y}{N_{0} \cdot \Delta F} \cdot \frac{K \cdot x+1}{1+x}\right)\right]_{(50)}^{K}$

Accordingly, the function $h(z)$ is defined as

$$
h(z)=1-\left[1-\frac{1}{2} \cdot \exp \left(-\frac{1}{2} \cdot \frac{A \cdot z}{N_{0} \cdot \Delta F}\right)\right]^{K}
$$

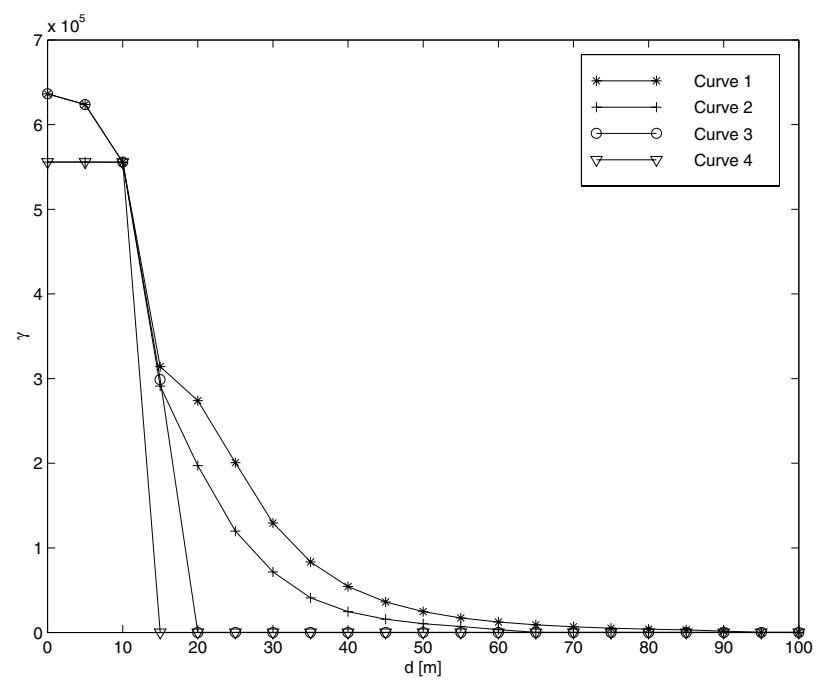

Fig. 9. Maximum satisfaction function, $\gamma$, vs. the distance $d$ between the transmitter and the receiver.

In Figure 9 we give the maximum values of the satisfaction function, $\gamma$, vs. the distance between the transmitter and the receiver. Also in this case, for the sake of comparison four curves are shown in Figure 9. Their meaning was described 
in Section IV-B. Again Figure 9 was obtained using the parameters given in Table I.

\section{Conclusions}

In this paper we have studied how forward error correction (FEC) protocols and power management can be utilized in conjunction to maximize TCP performance.

To this end we have introduced an analytical framework which can be used to determine the optimal values of the transmission power level, $y_{\mathrm{opt}}$, and the ratio of redundancy introduced by FEC, $x_{\mathrm{opt}}$. By definition $x_{\mathrm{opt}}$ and $y_{\mathrm{opt}}$ are the values which maximize a satisfaction function, $\gamma(x, y)$ defined as the ratio between the TCP throughput and the cost function. We have derived a relationship between $x_{\mathrm{opt}}$ and $y_{\mathrm{opt}}$ which is independent of the numerical modulation technique utilized and does not rely on any TCP throughput approximation formula.

The proposed analytical framework is applied to the cases of Gaussian M-ary Shift Keying (GMSK), Differentiated Binary Phase Shift Keying (DBPSK) and Gaussian Frequency Shift Keying (GFSK) which are used in very common wireless systems such as GPRS, IEEE 802.11 and Bluetooth. Our results show that by joint management of error control and transmission power, higher satisfaction function values can be achieved when compared to those obtained when error control and power management are considered separately.

\section{REFERENCES}

[1] E. Altman, C. Barakat and V. M. Ramos R. Queueing analysis of simple FEC schemes for IP Telephony. Proc. of IEEE Infocom 2001. Anchorage, Alaska. April 2001.

[2] A. Bakre and B. R. Badrinath. I-TCP: Indirect TCP for Mobile Hosts. Proc. of 15-th International Conference on Distributed Computing Systems (ICDCS). Vancouver, Canada. May 1995.

[3] H. Balakrishnan, S. Seshan, E. Amir and R. H. Katz. Improving TCP/IP Performance over Wireless Networks. Proc. of ACM Mobicom'95. Berkeley, California. November 1995.

[4] H. Balakrishnan, S. Seshan, and R. H. Katz. Improving Reliable Transport Protocol and Handoff Performance in Cellular Wireless Networks. ACM-Baltzer Wireless Networks Journal. Vol. 1, No. 4. pp. 469-481. December 1995.

[5] H. Balakrishnan, V. N. Padmanabhan, S. Seshan and R. H. Katz. A Comparison of Mechanisms for Improving TCP Performance over Wireless Links. IEEE/ACM Transactions on Networking. Vol. 5, No. 6. pp. 756-769. December 1997.

[6] C. Barakat and E. Altman. Bandwidth Trade-Off between TCP and LinkLevel FEC. Computer Networks. Vol. 39, No. 2. pp. 133-150. June 2002.

[7] C. Casetti, M. Gerla, S. Mascolo, M. Y. Sanadidi, R. Wang. TCP Westwood: Bandwidth Estimation for Enhanced Transport over Wireless Links. Proc. of ACM Mobicom 2001. Rome, Italy. July 2001.

[8] H.M. Chaskar, T.V. Lakshman, and U. Madhow. TCP Over Wireless with Link Level Error Control: Analysis and Design Methodology. IEEE/ACM Transactions on Networking. Vol. 7, No. 5. pp. 605-615. October 1999.

[9] S. Cho, A. Goulart, I. F. Akyildiz and N. Jayant. An Adaptive FEC with QoS Provisioning for Real-Time Traffic in LEO Satellite Networks. Proc. of IEEE ICC 2001. Helsinki, Finland. June 2001.

[10] S. Cho Adaptive Error Control Scheme for Multimedia Applications in Integrated Terrestrial-Satellite Wireless Networks. Proc. of IEEE WCNC 2000. Chicago, Illinois. September 2000.

[11] T. Dubendorf. 802.11 The Wireless LAN Whitepaper Series. Part I: Is it For You?. Lucent NPS Whitepaper, 2000.

[12] M. Handley, J. Padhye, S. Floyd, and J. Widmer. TCP Friendly Rate Control (TFRC): Protocol Specification. Internet Draft. draft-iet $\mathrm{f}$ tsvwg-tfrc-03.txt. July 2001
[13] V. Jacobson. Congestion Avoidance and Control. Proc. of ACM SIGCOMM'88. Stanford, California. August 1988

[14] V. Jacobson. Congestion Avoidance and Control. Technical Report. April 1990.

[15] T.V. Lakshman and U. Madhow. The Performance of TCP/IP for Networks with High Bandwidth-Delay Products and Random Loss. IEEE/ACM Transactions on Networking. Vol. 5, No. 3. pp. 336-350. June 1997.

[16] J. Padhye, V. Firoiu, D. Towsley, and J. Kurose. Modeling TCP Throughput: A Simple Model and its Empirical Validation. Proc. of ACM SIGCOMM 1998. Vancouver, Canada. October 1998.

[17] J.G. Proakis. Communication Systems Engineering. Prentice Hall International Editions. 1994.

[18] I.S. Reed and G. Solomon. Polynomial Codes Over Certain Finite Fields. Journal of the Society for Industrial and Applied Mathematics. Vol. 8, No. 4. pp. 300-304. June 1960.

[19] G. Xylomenos and G. C. Polyzos. Link Layer Support for Quality of Service on Wireless Internet Links. IEEE Personal Communications. Vol. 6, No. 5. pp. 52-60. October 1999.

[20] M. Zorzi, M. Rossi, G. Mazzini. Throughput and energy performance of TCP on a wideband CDMA air interface. Wireless Communications and Mobile Computing. Vol 2, No. 1. pp. 71-84, February 2002.

[21] http://grouper.ieee.org/groups/802/11/ 